\title{
Laparoscopy-assisted Proximal Gastrectomy with Double Tract Anastomosis Is Beneficial for Vitamin B12 and Iron Absorption
}

\author{
DONG JIN KIM and WOOK KIM \\ Department of Surgery, Yeouido St. Mary's Hospital, College of Medicine, \\ The Catholic University of Korea, Seoul, Republic of Korea
}

\begin{abstract}
Background: Double tract anastomosis (DTA) is a recently revisited reconstruction method for preventing reflux esophagitis in laparoscopy-assisted proximal gastrectomy (LAPG). However, only few studies have shown the advantages of LAPG-DTA. Patients and Methods: Seventeen patients underwent LAPG-DTA compared to 17 patients undergoing laparoscopy-assisted total gastrectomy (LATG) matched with preoperative stage. Laboratory results, including hemoglobin, serum ferritin, serum iron and vitamin B12, were compared. Results: Reflux esophagitis developed in two and one patient in the LAPG-DTA and LATG groups, respectively. In the laboratory analysis, fewer patients experienced decrease of serum ferritin below the low normal limit in the LAPG-DTA group. There was a significantly lower proportion of patients in the LAPG-DTA group whose vitamin B12 level decreased below the low normal limit. Conclusion: LAPG-DTA has the advantages of allowing absorption of iron and vitamin B12 without reflux esophagitis in proximal early gastric cancer compared to LATG.
\end{abstract}

According to a nationwide survey in Korea, the incidence of early gastric cancer (EGC) and proximal gastric cancer increased from $43.2 \%$ in 2004 to $57.6 \%$ in 2009 and $12.5 \%$ to $13.4 \%$, respectively $(1,2)$. Patients with EGC usually undergo total gastrectomy (TG), proximal gastrectomy (PG) is rarely performed (2). Traditionally, PG with esophagogastrostomy (EG) has been avoided due to the possibility of reflux esophagitis and anastomotic stricture. However, with the increased demand for less invasive surgical techniques and for maintenance of quality of life, laparoscopy-assisted

Correspondence to: Professor Wook Kim, MD, Ph.D., Division of Gastrointestinal Surgery, Department of Surgery, Yeouido St. Mary's Hospital, College of Medicine, The Catholic University of Korea, \#62 Yeouido-dong, Yeongdeungpo-gu, Seoul, 150-713, Korea. Tel: +82 237792020, Fax: +82 27860802, e-mail: kimwook@catholic.ac.kr

Key Words: Proximal gastrectomy, double tract anastomosis, vitamin B12. proximal gastrectomy (LAPG) has been revisited in clinical practice (3). To overcome anastomosis-related problems, various types of anastomosis have been introduced to avoid reflux esophagitis, one of which is double tract anastomosis (DTA) (4). DTA has been reported to have the advantage over traditional EG of a lower incidence of reflux esophagitis (3-5). However, other effects of DTA, including effects on anemia, iron metabolism, vitamin B12 level and nutritional parameters have rarely been reported. In this study, surgical outcomes and laboratory data following LAPG-DTA and laparoscopy-assisted total gastrectomy (LATG) were compared.

\section{Patients and Methods}

Patients. At our Institute, 73 patients with proximal gastric cancer underwent LATG $(n=46)$ or LAPG $(n=27)$ from April 2009 to December 2014. LAPG was performed for clinically T1N0M0 patients. Among the LAPG patients, 17 underwent LAPG-DTA from December 2011 to December 2014. To match indication for surgery, 17 patients with preoperative stage T1N0M0 who underwent LATG were selected for comparison with the LAPGDTA patients with regards to clinicopathological characteristics and surgical results. Among 17 patients in each group, 10 LAPG-DTA and 13 LATG patients were selected for evaluation of laboratory data, as at least 1 year of follow-up laboratory data were available for those patients. Laboratory results are presented with the number and proportion of patients whose laboratory values decreased under the lower normal limit.

Evaluation and postoperative management. All analyses were based on an institutional database that contained prospectively collected data. Sex, age, American Society of Anesthesiologists (ASA) physical status score, body mass index, tumor location, tumor size, tumor depth, node status, and final stage were collected. Surgical outcomes included operation time, hospital stay, number of retrieved lymph nodes, early complications (0-30 days), and late complications (>30 days), including reflux and anastomosis stricture. Early complications were only included for those with a Clavian-Dindo classification of IIIa or greater. Esophageal reflux was defined by clinical symptom and was managed with proton pump inhibitors. Stricture was defined as the need for balloon dilatation. Laboratory studies were performed every 6 months after 
the operation and included hemoglobin, serum iron, serum ferritin, vitamin B12, total protein, and albumin measurements. During the follow-up period, $1 \mathrm{mg}$ of cobamamide was supplied parenterally every 6 months after 1 year from surgery.

Operative procedures. LATG was performed according to previously described procedures, and esophagojejunostomy was performed intracorporeally using the OrVil ${ }^{\mathrm{TM}}$ system (Covidien, Mansfield, MA, USA) (6). The same trocar size and placement were used during LAPG-DTA as during LATG. The resection line was individualized based on the distal margin of the tumor. The extent of lymph node dissection for LAPG was D1+, according to the Japanese gastric cancer treatment guidelines (7). After stomach resection and lymph node dissection, the left upper quadrant trocar site was extended to $4-5 \mathrm{~cm}$ in length and was used for gastrojejunostomy (GJ) and jejunojejunostomy. Esophagojejunostomy was carried out intracorporeally using the OrVil ${ }^{\mathrm{TM}}$ system for both LAPG-DTA with LATG. The GJ was made at the alimentary limb, $15 \mathrm{~cm}$ distal of the esophagojejunostomy, and the jejunojejunostomy was made $25 \mathrm{~cm}$ distal to the GJ. For LAPGDTA, the final reconstruction is illustrated in Figure 1.

Statistical analysis. Categorical variables were compared using Pearson's Chi-square test, and continuous variables were compared using Student's $t$-test. All continuous variables are expressed as means \pm standard deviation. The statistical analyses were performed with SPSS statistical software version 18.0 for Windows (SPSS, Chicago, IL, USA).

\section{Results}

For the clinicopathological characteristics, there were no differences in sex, age, ASA score or body mass index between the two groups (Table I). Two and three patients with pathologic T2 disease were included in the LAPG-DTA and LATG groups, respectively. Most patients had stage I disease according to the seventh Union of International Cancer Control staging system (8), except for three patients with stage II disease (one in the LAPG-DTA group and two in the LATG group).

For the surgical results (Table II), there were no differences in operative time $(p=0.922)$ or hospital stay $(p=0.660)$. There were three major complications (ClavienDindo IIIa or greater) in both groups. In the LAPG-DTA group, pleural effusion with drainage catheter insertion, reoperation for intra-abdominal bleeding, and pneumonia requiring intensive care unit care occurred. There were two cases of reoperation for intra-abdominal bleeding and one of anastomotic leakage requiring for percutaneous drainage catheter insertion in the LATG group. With regards to longterm symptoms, two patients suffered from reflux esophagitis in the LAPG-DTA group at 2 years after surgery; however, it was well-managed with proton pump inhibitor administration for 1 month.

Laboratory data were available for 10 patients in the LAPG-DTA group and 13 patients in the LATG group (Table

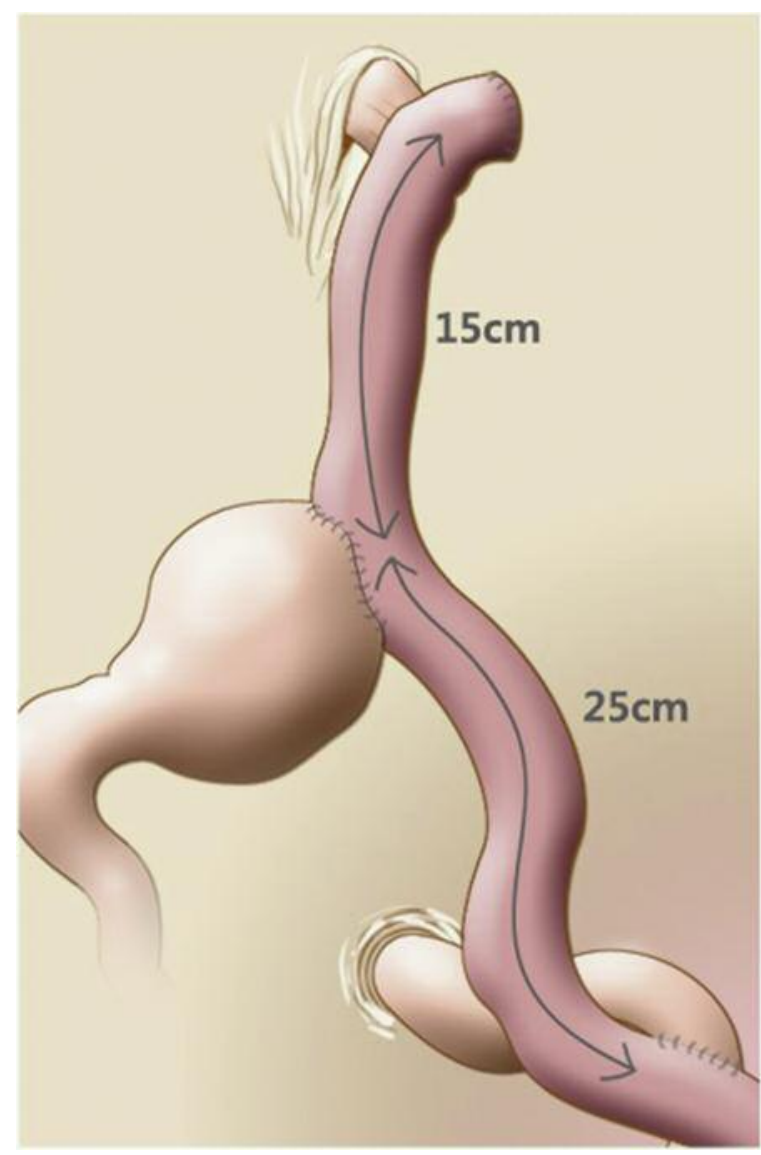

Figure 1. Illustration of laparoscopy-assisted proximal gastrectomy with double tract anastomosis.

III). More LATG patients experienced a decrease of serum ferritin level below the lower normal limit than did LAPGDTA patients, but this difference was not statistically significant (LAPG-DTA $v s$. LATG: $p=0.231$ ). For significantly more patients in the LATG group, the vitamin B12 level decreased below the lower normal limit $(p=0.027)$. In the serial comparison of anemia-related laboratory studies, only the vitamin B12 level was significantly different between the two groups from 18 months following surgery. Total protein and albumin levels were not different between the two groups (Figure 2).

\section{Discussion}

In this study, we compared surgical outcomes and laboratory changes between patients who underwent LAPG-DTA and LATG. LAPG-DTA had favorable results in terms of anastomosis-related complications and anemia-related parameters, especially regarding the vitamin B12 level. 
Table I. Clinicopathological features of all patients included in this study.

\begin{tabular}{|c|c|c|c|}
\hline & LAPG-DTA $(n=17)$ & LATG $(n=17)$ & $p$-Value \\
\hline \multicolumn{4}{|l|}{ Gender } \\
\hline Male & $14(82.4)$ & $10(58.8)$ & \multirow[t]{2}{*}{0.132} \\
\hline Female & $3(17.6)$ & 7 (41.2) & \\
\hline Age (years) & $64.7 \pm 9.9$ & $60.9+12.9$ & 0.178 \\
\hline \multicolumn{4}{|l|}{ ASA } \\
\hline 1 & $8(47.1)$ & $9(52.9)$ & \multirow[t]{3}{*}{0.265} \\
\hline 2 & $9(52.9)$ & $6(35.3)$ & \\
\hline 3 & 0 & $2(11.8)$ & \\
\hline BMI $\left(\mathrm{kg} / \mathrm{m}^{2}\right)$ & $24.2 \pm 3.8$ & $23.4+5.0$ & 0.541 \\
\hline \multicolumn{4}{|l|}{ Tumor location } \\
\hline Cardia & $8(47.1)$ & $8(47.1)$ & \multirow[t]{2}{*}{0.732} \\
\hline High body & $9(52.9)$ & $9(52.9)$ & \\
\hline Tumor size $(\mathrm{cm})$ & $2.2 \pm 1.0$ & $4.0+3.2$ & 0.060 \\
\hline \multicolumn{4}{|l|}{ Tumor depth } \\
\hline T1a & $6(35.3)$ & $8(47.1)$ & \multirow[t]{3}{*}{0.581} \\
\hline $\mathrm{T} 1 \mathrm{~b}$ & $9(52.9)$ & $6(35.3)$ & \\
\hline $\mathrm{T} 2$ & $2(11.8)$ & $3(17.6)$ & \\
\hline \multicolumn{4}{|l|}{$\mathrm{N}$-Stage } \\
\hline No & $14(82.4)$ & $15(88.2)$ & \multirow[t]{4}{*}{0.386} \\
\hline N1 & $2(11.8)$ & $0(0)$ & \\
\hline $\mathrm{N} 2$ & $0(0)$ & $1(5.9)$ & \\
\hline N3 & $1(5.9)$ & $1(5.9)$ & \\
\hline \multicolumn{4}{|l|}{ Tumor stage } \\
\hline Ia & $12(70.6)$ & $12(70.6)$ & \multirow[t]{4}{*}{0.767} \\
\hline $\mathrm{Ib}$ & $4(23.5)$ & $3(17.6)$ & \\
\hline IIa & $0(0)$ & $1(5.9)$ & \\
\hline IIb & $1(5.9)$ & $1(5.9)$ & \\
\hline
\end{tabular}

LAPG-DTA: Laparoscopy-assisted proximal gastrectomy with double tract anastomosis; LATG: laparoscopy-assisted total gastrecomy; ASA: American society of anesthesiologists physical status score; BMI: body mass index. Continuous variables are expressed as means \pm standard deviation, and nominal variables are expressed as numbers of patients (percentages).

Although survival is not different following PG and TG, in patients with upper EGC, PG is traditionally avoided due to anastomosis-related complications $(5,9)$. In order to overcome this problem, many alternative procedures have been performed, such as jejunal interposition, gastric tube EG, lower esophageal sphincter-preserving EG, and DTA (10-13). However, the results of studies of these procedures have been inconsistent, and most studies have been retrospective. No large-scale randomized controlled studies have been performed. Therefore, currently, there is no optimal reconstruction method for PG.

Recent advances in laparoscopic surgery have allowed various function-preserving surgeries to be performed. LAPG is one minimally invasive form of surgery that has gained attention, and more simple reconstruction methods suitable for laparoscopic surgery are needed. DTA was originally designed to allow for smooth transfer of larger
Table II. Surgical results in both groups in this study.

\begin{tabular}{lccc}
\hline Variable & LAPG-DTA (n=17) & LATG $(\mathrm{n}=17)$ & $p$-Value \\
\hline Operative time (min) & $268.2 \pm 40.9$ & $270.2 \pm 43.4$ & 0.922 \\
Hospital stay (days) & $9.4 \pm 4.1$ & $10 \pm 3.6$ & 0.660 \\
Retrieved lymph nodes & $33.4 \pm 17.3$ & $39.3 \pm 20.4$ & 0.384 \\
Early complication* & $3(17.6)$ & $3(17.6)$ & 0.169 \\
$\quad$ Dindo IIIa & $1(5.9)$ & $1(5.9)$ & \\
$\quad$ Dindo IIIb & $1(5.9)$ & $2(11.8)$ & \\
$\quad$ Dindo IVa & $1(5.9)$ & $0(0)$ & \\
Anastomosis-related & & & \\
complication & $2(11.8)$ & $1(5.9)$ & 0.545 \\
$\quad$ Reflux & $0(0)$ & $1(5.9)$ & 1.000 \\
$\quad$ Stricture & & & \\
\hline
\end{tabular}

LAPG-DTA: Laparoscopy-assisted proximal gastrectomy with double tract anastomosis; LATG: laparoscopy-assisted total gastrecomy. Continuous variables are expressed as means \pm standard deviation, and nominal variables are expressed as numbers of patients (percentages). *LAPG-DTA: IIIa: pleural effusion-drainage, IIIb: intra-abdominal bleeding, Iva: pneumonia; LATG: IIIa: anastomotic leakage, IIIb: both intra-abdominal bleeding.

Table III. Numbers and proportion of patients whose laboratory values decreased below the lower normal limit.

\begin{tabular}{lccc}
\hline $\begin{array}{l}\text { Variable } \\
\text { (normal range) }\end{array}$ & $\begin{array}{c}\text { LAPG-DTA } \\
(\mathrm{n}=10)\end{array}$ & $\begin{array}{c}\text { LATG } \\
(\mathrm{n}=13)\end{array}$ & $p$-Value \\
\hline $\begin{array}{l}\text { Iron }(60-200 \mu \mathrm{g} / \mathrm{dl}) \\
\quad \text { Normal }\end{array}$ & $10(100)$ & $11(84.6)$ & 0.194 \\
$\quad$ Below normal & $0(0)$ & $2(15.4)$ & \\
Ferritin $(22-322 \mathrm{ng} / \mathrm{ml})$ & $9(90.0)$ & $9(69.2)$ & 0.231 \\
$\quad$ Normal & $1(10.0)$ & $4(30.8)$ & \\
$\quad$ Below normal & & & \\
Vitamin B12 $(211-911 \mathrm{pg} / \mathrm{ml})$ & $10(100)$ & $8(61.5)$ & 0.027 \\
$\quad$ Normal & $0(0)$ & $5(38.5)$ & \\
$\quad$ Below normal & & & \\
\hline
\end{tabular}

LAPG-DTA: Laparoscopy-assisted proximal gastrectomy with double tract anastomosis; LATG: laparoscopy-assisted total gastrecomy. All data are expressed as numbers of patients (percentages).

foods through the duodenal route and was first reported in 1988 by Aikou et al. (14). Recently, DTA in conjunction with laparoscopy has become popular among some gastric surgeons and has shown favorable results regarding anastomosis-related complications and functional outcomes $(3,4,6,15)$.

However, the benefits of this alternative procedure over the conventional one should be proven before it is generally accepted. Therefore, we performed this comparative study. Theoretically, there are two definite advantages of DTA 

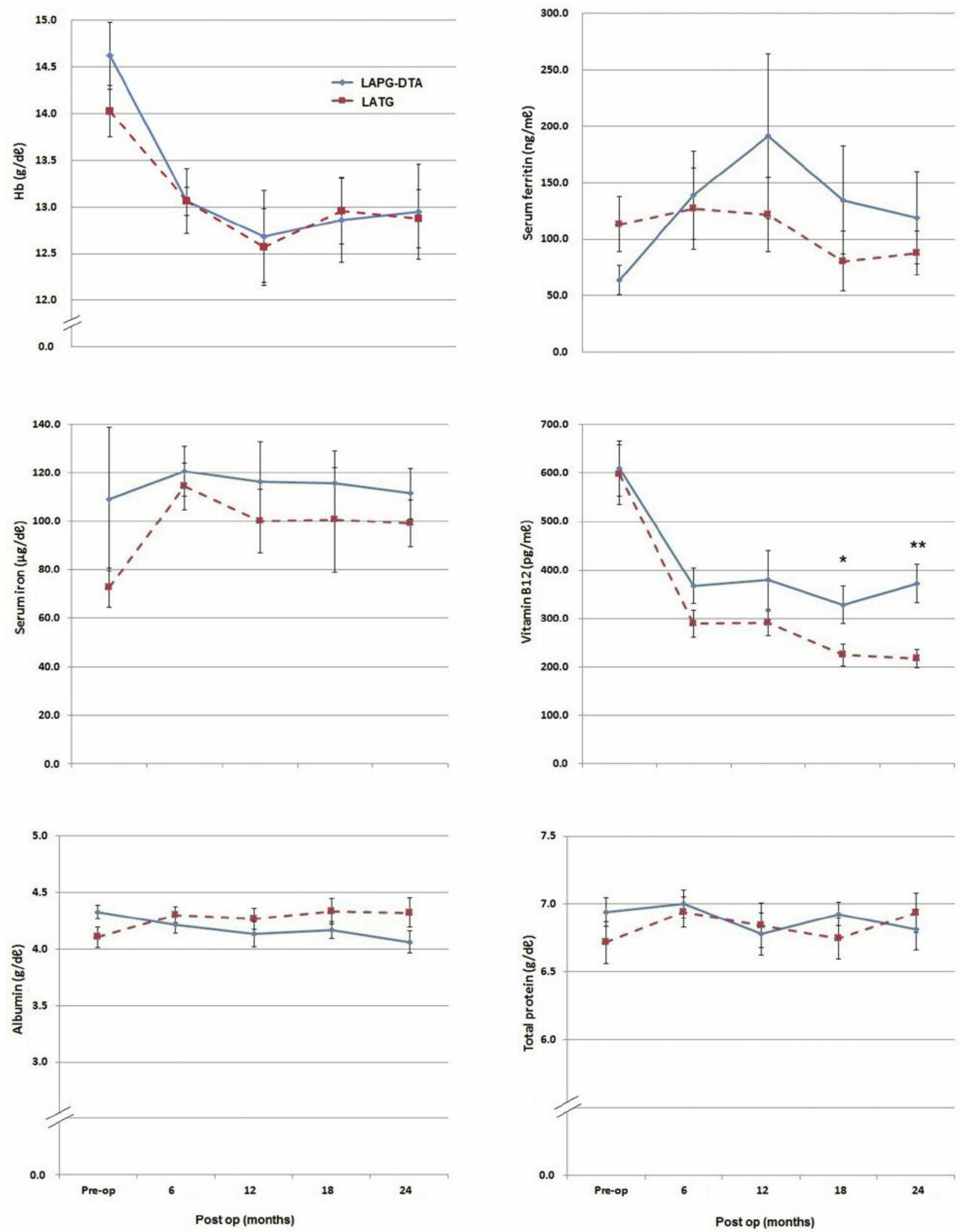

Figure 2. Serial laboratory findings for laparoscopy-assisted proximal gastrectomy with double tract anastomosis (LAPG-DTA) and laparoscopyassisted total gastrectomy (LATG) groups. Hb: Hemaglobin. Between-group comparison: * $p=0.025$ and **p=0.002. 
compared to TG. One is that food passage through both the jejunal alimentary limb and through the remnant stomach to the duodenum can be achieved. In fact, an upper GI study revealed bidirectional passage, which contrasted with the findings of our study (Figure 3). In addition, Ahn et al. performed a gastric-emptying study with $2 \mathrm{mCi}$ technetium$99 \mathrm{~m}$-diethylene-triamine-penta-acetate and found that the relative ratio of food flow from the stomach to the small intestine was 3:2 (4). Bypassing the duodenum and proximal jejunum is related to iron absorption, which occurs in the duodenum (16). Laboratory data regarding iron absorption have been rarely reported by other comparative studies of TG and PG $(5,9,17)$. In the current study, more patients experienced a decreased serum ferritin level below the lower normal limit in the LATG group than in the LAPG-DTA group, but this difference was not statistically significant. Although the hemoglobin level was not different between the two groups, our data indicate an effect of DTA on ferritin level. A previous study found evidence of benefits of DG over GJ with regards to prevention of iron deficiency in patients undergoing DG (18). The main difference in the results of these two procedures was whether or not food passed through the duodenum. As decreases in serum ferritin levels are the first manifestation of iron deficiency anemia, the difference in the extent of resection and anastomosis between the two groups may affect iron metabolism.

LAPG-DTA preserves the gastric antrum and part of the gastric body, allowing for food reserve, which indicates that these areas of the stomach are potential sources of intrinsic factors. In our study, there were significant advantages to LAPG-DTA with regards to vitamin B12 absorption starting at 18 months postoperatively. TG is known to have a risk of leading to vitamin B12 deficiency compared to DG (19). Because the main source of intrinsic factors in the stomach are parietal cells, which are mainly located in the body and fundus, the ability of PG to prevent vitamin B12 deficiency is questionable. In gastrectomized patients, we evaluated the vitamin B12 level every 6 months and supplied vitamin B12 parenterally every 6 months after 1 year from surgery because both groups underwent resection of the gastric body and fundus, which are the main source of intrinsic factors Even with regular vitamin B12 injection, patient of the LATG group had significantly lower levels of vitamin B12, which is the proof of maintained function of remnant stomach in the LAPG group. Recent long-term results of open TG and PG showed a prominent advantage of PG with regards to vitamin B12 metabolism (17). Those studies showed sufficient maintenance of antral function with regards to vitamin B12 absorption.

Food reserve and physiological nutrient passage are important for nutritional support. However, we failed to show superiority of LAPG-DTA with regards to nutritional parameters, such as albumin and total protein levels.

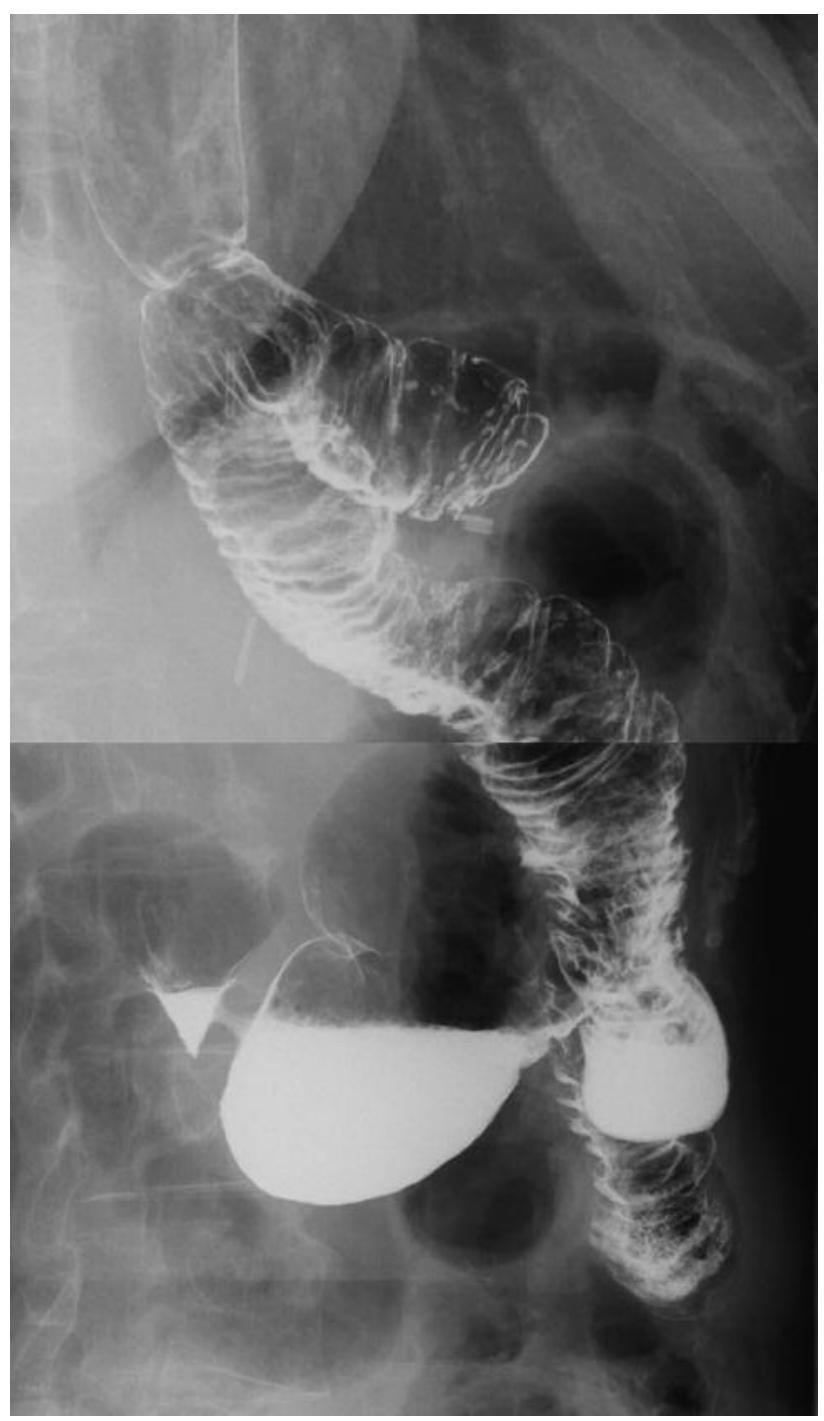

Figure 3. Upper gastrointestinal barium swallowing test shows dual pathway to distal stomach and jejunum.

With regards to anastomosis and surgical safety, GJ is formed in LAPG-DTA instead of maintaining the duodenal stump. Generally, GJ has fewer complications than duodenal stump mainly because it generates a sufficient blood supply. In our previous study, duodenal stump more frequently caused complications than GJ (20). Although there were no differences between the two groups in the rate of leakage in this study, surgeons can ensure duodenal stump leakage will not occur by forming a DTA.

There are two problems with DTA. The first is confirming that food passage through the duodenum is occurring. Although a contrast study revealed food passage through the duodenum and a gastric-emptying scan showed that $60 \%$ of 
ingested foods were in the remnant stomach, the passage of food material through the duodenum is not clear. It can differ according to whether the food is liquid or solid. Food passage through the duodenum needs to be shown to confirm the theoretical advantages of LAPG-DTA.

Another issue is surveillance of the remnant stomach. Because the distal esophagus and remnant stomach are connected with a $10-15 \mathrm{~cm}$ section of the jejunum, endoscopic observation of the remnant stomach is possible. However, a flexible scope is not easily adjustable when it enters the remnant stomach through the jejunum. In addition, making a U-turn with a flexible scope to view the anastomosis site from the remnant antral side is troublesome in some patients.

In conclusion, LAPG-DTA is beneficial with regards to the absorption of iron and vitamin B12 compared to LATG without increasing reflux esophagitis. LAPG-DTA is a good alternative procedure for the treatment of proximal EGC.

\section{References}

1 The Information Committee of the Korean Gastric Cancer Association: 2004 Nationwide Gastric Cancer Report in Korea. J Korean Gastric Cancer Assoc 7(1): 47-54, 2007.

2 Jeong $\mathrm{O}$ and Park YK: Clinicopathological features and surgical treatment of gastric cancer in South Korea: the results of 2009 nationwide survey on surgically treated gastric cancer patients. J Gastric Cancer 11(2): 69-77, 2011.

3 Nomura E, Lee SW, Kawai M, Yamazaki M, Nabeshima K, Nakamura $\mathrm{K}$ and Uchiyama K: Functional outcomes by reconstruction technique following laparoscopic proximal gastrectomy for gastric cancer: double tract versus jejunal interposition. World J Surg Oncol 12: 20, 2014.

4 Ahn SH, Jung do H, Son SY, Lee CM, Park do J and Kim HH: Laparoscopic double-tract proximal gastrectomy for proximal early gastric cancer. Gastric Cancer 17(3): 562-570, 2014.

5 Ahn SH, Lee JH, Park do J and Kim HH: Comparative study of clinical outcomes between laparoscopy-assisted proximal gastrectomy (LAPG) and laparoscopy-assisted total gastrectomy (LATG) for proximal gastric cancer. Gastric Cancer 16(3): 282$289,2013$.

6 Jung YJ, Kim DJ, Lee JH and Kim W: Safety of intracorporeal circular stapling esophagojejunostomy using trans-orally inserted anvil (OrVil) following laparoscopic total or proximal gastrectomy - comparison with extracorporeal anastomosis. World J Surg Oncol 11: 209, 2013.

7 Japanese Gastric Cancer Association: Japanese gastric cancer treatment guidelines 2010 (ver. 3). Gastric Cancer 14(2): 113123, 2011.

8 Kwon SJ: Evaluation of the seventh UICC TNM Staging System of Gastric Cancer. J Gastric Cancer 11(2): 78-85, 2011.
9 Wen L, Chen XZ, Wu B, Chen XL, Wang L, Yang K, Zhang B, Chen ZX, Chen JP, Zhou ZG, Li CM and Hu JK: Total vs. proximal gastrectomy for proximal gastric cancer: a systematic review and meta-analysis. Hepatogastroenterology 59(114): 633640, 2012.

10 Hirai T, Matsumoto H, Iki K, Hirabayashi Y, Kawabe Y, Ikeda M, Yamamura M, Hato S, Urakami A, Yamashita K, Tsunoda T and Haruma K: Lower esophageal sphincter- and vaguspreserving proximal partial gastrectomy for early cancer of the gastric cardia. Surg Today 36(10): 874-878, 2006.

11 Nakamura $M$ and Yamaue $H$ : Reconstruction after proximal gastrectomy for gastric cancer in the upper third of the stomach: a review of the literature published from 2000 to 2014. Surg Today 46(5): 517-527, 2016.

12 Sakuramoto S, Yamashita K, Kikuchi S, Futawatari N, Katada $\mathrm{N}$, Moriya H, Hirai K and Watanabe M: Clinical experience of laparoscopy-assisted proximal gastrectomy with Toupet-like partial fundoplication in early gastric cancer for preventing reflux esophagitis. J Am Coll Surg 209(3): 344-351, 2009.

13 Zhao P, Xiao SM, Tang LC, Ding Z, Zhou X and Chen XD: Proximal gastrectomy with jejunal interposition and TGRY anastomosis for proximal gastric cancer. World J Gastroenterol 20(25): 8268-8273, 2014.

14 Aikou T, Natsugoe S, Shimazu H and Nishi M: Antrum preserving double tract method for reconstruction following proximal gastrectomy. Jpn J Surg 18(1): 114-115, 1988.

15 Nakajima K, Kawano M, Kinami S, Fujimura T, Miwa K and Tonami N: Dual-radionuclide simultaneous gastric emptying and bile transit study after gastric surgery with double-tract reconstruction. Ann Nucl Med 19(3): 185-191, 2005.

16 Fleming RE and Bacon BR: Orchestration of iron homeostasis. N Engl J Med 352(17): 1741-1744, 2005.

17 Son MW, Kim YJ, Jeong GA, Cho GS and Lee MS: Long-term outcomes of proximal gastrectomy versus total gastrectomy for upper-third gastric cancer. J Gastric Cancer 14(4): 246-251, 2014.

18 Lee JH, Hyung WJ, Kim HI, Kim YM, Son T, Okumura N, Hu Y, Kim CB and Noh SH: Method of reconstruction governs iron metabolism after gastrectomy for patients with gastric cancer. Ann Surg 258(6): 964-969, 2013.

$19 \mathrm{Hu}$ Y, Kim HI, Hyung WJ, Song KJ, Lee JH, Kim YM and Noh SH: Vitamin B(12) deficiency after gastrectomy for gastric cancer: an analysis of clinical patterns and risk factors. Ann Surg 258(6): 970-975, 2013.

$20 \mathrm{Kim}$ DJ, Lee JH and Kim W: Comparison of the major postoperative complications between laparoscopic distal and total gastrectomies for gastric cancer using Clavien-Dindo classification. Surg Endosc 29(11): 3196-3204, 2015.

Received June 30, 2016

Revised July 14, 2016

Accepted July 15, 2016 